\title{
'Treating a patient should be approached in a holistic manner': collaboration of doctors and physiotherapists in the rehabilitation of people living with HIV
}

\author{
Stacy Maddocks (D), Verusia Chetty* (D), Arishna Maghoo, Nkuleleko Mhlongo, Nsindiso Mthembu, Sinempilo Khanyile, Siphokazi \\ Chiliza, Tyrel Munsamy, Zanele Gamede and Slindile Mazibukoc ${ }^{a}$
}

Physiotherapy, School of Health Sciences, University of KwaZulu-Natal, Westville, Durban, South Africa

*Corresponding author, email:chettyve@ukzn.ac.za

People living with HIV facing impairments and subsequent disabilities related to the virus and its treatment require involvement of a collaborative team of healthcare professionals to ensure reintegration into daily life and community living. Healthcare teams responsible for this care include doctors and physiotherapists. This paper explores the collaboration of doctors and physiotherapists in the rehabilitation of people living with HIV in a semi-rural healthcare setting in KwaZulu-Natal, South Africa. Six doctors and two physiotherapists were interviewed using a semi-structured interview guide. The qualitative approach led to the emergence of five themes, namely a biomedical versus biopsychosocial approach; scope of practice challenge; multidisciplinary team enigma; institutional structure limitations; and recommendations from healthcare professionals. Both groups of professionals believed that a lack of understanding of the scope of practice and role of the associate profession in the multidisciplinary team led to poor referrals and lack of communication. Furthermore, shortage of personnel and resource limitations posed barriers to effective team interaction. Timely referrals, good communication and understanding of roles were suggested as endorsements to improved collaboration.

Keywords: Collaboration, doctors, HIV, multidisciplinary, physiotherapists, rehabilitation

\section{Introduction}

The HIV pandemic is still rampant, with approximately 37 million people living with the virus around the world. ${ }^{1}$ In sub-Saharan Africa alone about 25 million people are infected and South Africa remains the epicentre of the virus. ${ }^{2}$ KwaZulu-Natal, an eastern coastal province in South Africa, has the highest number of people living with HIV (PLHIV). ${ }^{2}$ The introduction of antiretroviral therapy (ARTs) into the South African health system has significantly reduced the mortality rate of PLHIV, which means people live longer but experience comorbidities associated with the virus and its treatment. ${ }^{3}$ The impairments and disabilities associated with chronic conditions such as HIV require a core team of rehabilitation professionals to restore function and integrate individuals back into the communities they inhabit. ${ }^{4}$ These rehabilitation professionals, namely physicians, nurses, physiotherapist, dietitians, clinical psychologists and occupational therapists, form part of a multidisciplinary team (MDT) who are designated to work together collaboratively in order to offer optimum care to PLHIV. ${ }^{5}$ Doctors and physiotherapists are part of the core team responsible for the care of PLHIV; however, little is known about the collaboration between these professions. A study conducted by Chetty and Maharaj ${ }^{4}$ explored the relationship between the physiotherapist and the nurse in an urban context in South Africa. Inhibitors to collaboration included role governance, environmental structure and organisational variance. The authors suggested that education through mediums such as inservice training could contribute to improved teamwork. ${ }^{4}$ In another study investigating conflict within interprofessional primary healthcare teams, healthcare professionals believed that sources of conflict arose from role-boundary challenges and scope-of-practice dilemmas. Again, hierarchical structures within primary healthcare teams posed barriers to conflict resolution and this subsequently impedes collaboration of multidisciplinary teamwork. ${ }^{6}$

Primary care doctors and nurses are usually the first-line practitioners attending to patients within the South African healthcare system, and doctors are reported as being the dominant team members in the MDT.? They offer biomedical intervention and refer individuals-for the purposes of this paper PLHIV - to allied professions including physiotherapy. Physiotherapists are involved in the rehabilitation of physical impairments, activity limitations and participation restrictions related to the effects of the $\mathrm{HI}$ virus and its treatment. ${ }^{8}$ While the physiotherapist is crucial to re-integration of PLHIV into communities, the partnership between the doctor and the physiotherapist is vital for continuity of care. Optimal communication and understanding of the roles and scope of practice of each professional is important for cohesive collaboration; furthermore it is important to identify inhibitors to interdisciplinary partnership in order to offer solutions for improved teamwork. ${ }^{6}$ The study aimed to explore the collaboration between doctors and physiotherapists in the rehabilitation of PLHIV in a semi-rural setting in KwaZulu-Natal, South Africa, in order to offer perspectives on facilitators, inhibitors and suggested recommendations for a more holistic approach to patient care in this population.

\section{Methods}

A descriptive qualitative design using semi-structured interviews with doctors and physiotherapists was used to gather rich data from the healthcare professionals. The approach allowed for exploration into the lived experiences of the participating healthcare professionals. ${ }^{9}$ It allowed researchers to delve into the partnership between physiotherapists and doctors in addressing the health care of PLHIV. 


\section{Study setting}

The study setting was a hospital in eThekwini offering healthcare to approximately 4500 PLHIV. ${ }^{10}$ This setting has been the foundation for collaborative work on HIV and rehabilitation for a number of projects. ${ }^{11}$

\section{Participant recruitment}

Ethical clearance was obtained from the University of KwaZuluNatal and permission was granted from the relevant hospital authorities. Following approval, a maximum variation purposive sampling was used to recruit professionals. Doctors and physiotherapists employed at the study setting were invited to participate. Health professionals volunteered and no incentives were negotiated or offered.

\section{Data collection}

Semi-structured interviews were conducted with doctors and physiotherapists. The interview questions explored the collaborative practices between doctors and physiotherapists in managing PLHIV and included participants' views on their role and scope of practice in the rehabilitation of PLHIV, knowledge of HIV-related impairments, perceived challenges or barriers to effective collaboration, current referral practices and their recommendations for improved collaboration. Interviews were conducted in a private venue at the research setting according to the availability of the participants until no new information emerged and data saturation was achieved. Healthcare professionals were briefed on the procedure; two researchers conducted the interviews whilst another noted non-verbal cues of the participant responses. Interviews lasted for $20 \mathrm{~min}$ and were digitally recorded. Recorded data were stored electronically in a password-protected file.

\section{Data analysis}

Recorded data from the interviews were transcribed verbatim and analysed by the researchers. A stepwise approach to thematic data analysis was used to assimilate themes. ${ }^{12}$ The narratives from interviews were read repeatedly and common themes were extracted, distinguishing between similarities and differences. The data were given to an external auditor (peer reviewer) to review in order to maintain trustworthiness. Thick rich descriptions describing the setting, participants and detailed accounts of the narrative were implemented to maintain

Table 1: Themes and subthemes

\begin{tabular}{ll}
\hline Theme & Sub-themes \\
\hline $\begin{array}{l}\text { Biomedical versus biopsychosocial } \\
\text { approach }\end{array}$ & $\begin{array}{l}\text { Unseen complications of HI virus } \\
\text { Side effects of HIV treatment } \\
\text { Psychological impact }\end{array}$ \\
\hline Scope of practice challenge & $\begin{array}{l}\text { Physiotherapists' role according to } \\
\text { doctors }\end{array}$ \\
Doctors' role according to physiother- \\
apist
\end{tabular}

credibility. ${ }^{13}$ Pseudonyms were used when reflecting illustrative quotes from participants.

\section{Results}

Six doctors and two physiotherapists participated in the study. One physiotherapist was female, African aged 30 and the other male, Indian aged 27. There were three female and three male doctors who were aged between 30 and 57 years and who were all African and Indian. Their work experience ranged from six to 20 years in public healthcare practice.

Five themes arose from the interviews with the doctors and physiotherapists, namely a biomedical versus biopsychosocial approach, scope of practice challenge, multidisciplinary team enigma, institutional structure limitations, and recommendations for improved collaborative practice. Table 1 reflects the themes and subthemes from the interviews.

The theme biomedical versus biopsychosocial approach reflects the paradigm shift in viewing the virus as an impairment to a more holistic biopsychosocial condition. The subthemes 'unseen complications of the HI virus' and 'side effects of HIV treatment' reiterate that there is more to the virus than merely a'dysfunction of body systems' Quotes below emphasise the theme and the shift in view of the participating professionals.

Physiotherapist Tammy: 'HIV is not so much disabling but it's the complications that arise from it and its meds [referring to treatment] that render people to be disabled; peripheral neuropathy is one of them, HIV-related stroke or encephalopathy.

Doctor Ngcobo: 'Disability is a limitation of function in a person; in association with HIV it is the cause of the virus which can result in physical, psychological impairments and other conditions.

The second theme, scope of practice challenge. and subthemes of perceived roles of each profession emphasises the lack of understanding of healthcare professionals regarding their place within the multidisciplinary team. The quotes reflect the belief of the participants and professionals, who clearly iterated that they do not have adequate knowledge of the scope of practice of the interdisciplinary profession.

Doctor Ashen:'As doctors we do not know the full and appropriate treatments that physios provide there, we might not be referring enough patients, this might be caused due to our lack of exposure and knowledge about physiotherapy.'

Doctor Simphiwe: 'Physiotherapists mostly intervene with bedridden patients, those with muscular dystrophy, in order to help achieve their functional level, mobilisation being the longterm goal to be achieved.'

Physiotherapist Zando: 'They refer, we treat, I don't know everything they offer and they don't know everything I do.'

The theme 'multidisciplinary team enigma' arose from the medical hegemony and untimely and inappropriate referrals that physiotherapists alluded to and doctors corroborated in their interviews. In the narrative, one of the doctors displayed the dominant role as the gatekeeper to appropriate allied health care while another doctor believed that a good relationship was referral and allied health professions managing the referred patient. 
Doctor Thavi: 'Well, normally doctors are the first point of entry so the role of a doctor is to treat what they find and also refer to the appropriate departments where nutritional dietitian or physical therapists or whatever department it is the doctor that needs to make sure that the patient has access to all of those.'

Doctor Ngcobo: 'Well for me, I have a good relationship with the MDT, I refer to them and they do their treatments.'

The physiotherapy team felt the referral pathway was not adequate and this could be attributed to a lack of understanding of roles and scope of practice and is reflected in Doctor Simphiwe's quote.

Physiotherapist Tammy: 'you see because sometimes they don't refer'.

Doctors Simphiwe: 'The other challenge we face as doctors is inappropriate referrals to physio for such conditions; we are not sure whether physiotherapy treatment will be effective or not.'

The fourth theme of institutional structure limitations reflects the challenges around shortage of staff and scarcity of resources. The illustrative quotes encompass these beliefs.

Doctor Thando: 'It's sort of a challenge because the number of physiotherapists we have is not enough compared to the population of HIV patients we treat; one of the main challenges is the shortage of staff and the space to treat them.

Doctor Thavi:'Because of failure of getting everyone [referring to the multidisciplinary team] together, we always end up cancelling the meetings.'

Physiotherapist Tammy: 'Sometimes you can't even discuss the patients you see because sometimes if you not sure you have to look for that doctor and that is not always possible as we are short staffed and they are short staffed.'

Finally, the recommendations made included improved communication, education through in-service training and timely referrals. Supporting quotes from both professional groups are given below.

Doctor Ngcobo: 'Treating a patient should be approached in a holistic manner, therefore appropriate referral should be done by including other health members in the management of people living with HIV.'

Doctor Thando: 'Even with the doctors that are referring when they are communicating with the other members [referring to the MDT] they get to learn new things that can be done for patients therefore it increases their knowledge of what can be used for new patients with the same problems.'

Physiotherapist Zando: 'Whereas if it is in-service training where you are discussing then it is easy to know what is the way forward and to discuss what each of us does.'

\section{Discussion}

The number of doctors and physiotherapists employed at the facility was a good representation of the structures within a public healthcare system with scarcity of rehabilitation personnel. There were only two physiotherapists offering care to the catchment area, which includes several outlying clinics. ${ }^{10}$ In Cobbing et al., the lack of physiotherapists was attributed to availability of posts within the healthcare facilities in South Africa. The authors suggest that a proper analysis of the burden of disease within the country's health system needs to be conducted in order for a more comprehensive allocation of critical medical posts. ${ }^{8}$

Doctors and physiotherapists agreed that the effects of HIV and its treatment go beyond impairments and often impact on an individual's livelihood as well as social and community life. The realisation is in keeping with the current view of disability as described by the International Classification of Function, Disability and Health (ICF), ${ }^{14}$ which understands the multiple effects of disability. The ICF describes disability as not merely a consequence of disease and impairments but also as hindering functional activity and participation within the community bearing in mind that psychological and environmental factors could also influence disability. ${ }^{14,15}$ A study involving medical students' familiarity with the ICF framework directly influenced their management approaches to case-based scenarios in that familiarity contributed to a biopsychosocial approach to care compared with a biomedical slant. ${ }^{16}$ In 2013 , Hanass-Hancock et al. ${ }^{3}$ reported that the approach to HIV management is still very medical with little or no rehabilitation opportunity afforded to patients who need it. Similarly, a number of other studies have also identified the critical value of rehabilitation teams in managing the disability associated with HIV infection and treatment. ${ }^{4,8,17}$

The lack of understanding of respective professions was an overarching theme. Unfortunately, poor knowledge of professional roles is a common cause for poor team efficacy and reduced patient outcomes. ${ }^{18}$ Bechard (2010) believed that a contributing factor to lack of knowledge could be poor exposure to the scope of practice in undergraduate training. ${ }^{16}$ Chetty and Maharaj suggest that misunderstanding by the professional of roles within teams may lead to a professional divide and team dysfunction. ${ }^{4}$ Effective multidisciplinary interaction occurs when professionals are able to demonstrate knowledge of the practice of other professions within the team. ${ }^{19}$ An immediate effect of poor knowledge of the scope of practice is untimely and inappropriate referral trends, as evidenced in other studies. ${ }^{4}$ Interprofessional undergraduate education would again offer amelioration of such practice gaps. ${ }^{20}$ Realigning undergraduate health science curricula to include comprehensive interprofessional education in a South African context would also improve the multidisciplinary team dynamic. ${ }^{21}$

Hegemony is a barrier to collaboration as a dominant profession will impose decisions and this limits teamwork. ${ }^{7}$ In our study the medical professionals seemed to take the lead in the team and subsequent rehabilitative care proceeded after their assessment. Physiotherapists are first-line practitioners but within a South African healthcare system it is common practice that rehabilitation ensues following assessment by a doctor of physician. ${ }^{8}$ Similar findings were extracted from Baxter ${ }^{7}$ where power, status and decision-making belonged exclusively to doctors. ${ }^{7}$ Hierarchical dominance was also highlighted in a study in which a participant reported observing a medical resident openly scorning a nurse on the ward, demonstrating the power imbalance between doctors and the rest of the team. ${ }^{22}$ Teamwork requires respect and valuable, equal input from all members and this should be the norm within multidisciplinary teams for optimal care of individuals. ${ }^{6}$ The divide between professionals is 
often referred to as 'Tribalism' and results in distinctiveness amongst healthcare professionals. ${ }^{7}$ These distinctions need to be addressed in order to offer a holistic approach to health and rehabilitative care of a population of individuals with dynamic healthcare needs. ${ }^{6}$

Untimely and inappropriate referrals hindered optimal team collaboration. Inappropriate referrals are defined as those referrals for which physiotherapy is contraindicated and referrals that are not issued within the correct period for physiotherapy management to commence. ${ }^{23}$ Inappropriate referrals undermine the effectiveness of collaborative healthcare services resulting in poor patient outcomes, which includes the demise of some patients. Furthermore, poor patient outcomes result in negative attitudes and perceptions of health professions by patients and influence further care. ${ }^{18}$

The institutional structure was significant in the team dynamic as shortage of staff and scarcity of resources influenced collaboration. The shortage of physiotherapy staff affected doctors' decisions to refer PLHIV for rehabilitation. The physiotherapists in our study felt that that their interaction with doctors was equally affected due to their limited availability. Furthermore, participants reported that the lack of personnel from respective professions has resulted in the cessation of multidisciplinary ward meetings. Similarly Chetty and Maharaj ${ }^{4}$ evidenced that time constraints experienced by physiotherapists acted as a barrier to effective team collaboration in caring for PLHIV. Nurses in their study believed that the lack of time prevented physiotherapists from attending multidisciplinary ward rounds. ${ }^{4}$

Recommendations made by participants included improved communication, education and timely referral. Effective collaboration is based on optimal communication and understanding of individuals' role in the multidisciplinary team. ${ }^{17}$ The lack of communication creates a platform for compromised teamwork and patient outcomes as well as quality of care. ${ }^{10}$ Participants supported education such as in-service training as a forum to learn about the scope of respective professional practice. However, other researchers support interprofessional education at an undergraduate level as a possible solution to curb the knowledge divide. ${ }^{20}$ In Bechard's study the use of common assessment tools like the ICF was suggested as a means to bridge the knowledge and communication gap between health professionals and improve interdisciplinary collaboration and success. Authors believed that the biopsychosocial paradigm of the ICF would urge users to understand the holistic need of individuals such as PLHIV as in this paper. ${ }^{16}$ Finally, appropriate referrals issued in the correct timeframe for rehabilitation and when indicated will further team collaboration..$^{24}$ In the context of healthcare, collaboration amongst healthcare professionals is a catalyst for improved patient outcomes such as reduced hospital stay, costs and mortality rates as well as influencing patient satisfaction rates. ${ }^{25,26}$

\section{Conclusion}

The rehabilitative care offered to PLHIV needs to be collaborative and coordinated and to involve a team of skilled and knowledgeable healthcare professionals., ${ }^{4,5}$ Doctors and physiotherapist are significant members of the team responsible for the management of impairments related to HIV., ${ }^{4,78}$ Cohesive and proficient team interaction would significantly enhance the standard of care offered to PLHIV $4,7,8,26$ This study explored the collaboration between doctors and physiotherapists in the management of PLHIV at a semi-rural setting in South Africa. Effective partnership is necessary to facilitate good referral practices between healthcare professionals but is often a challenge to coordinate effectively in healthcare systems. ${ }^{18,24} \mathrm{~A}$ lack of insight into the scope of practice poses barriers to teamwork. Furthermore, staff shortages and resource limitations also hinder collaboration in healthcare team interaction. Improved communication and understanding as well as knowledge regarding roles and practice offers a solution to improved team dynamics. Interprofessional education is theoretically a possible answer to address the professional divide and 'tribalism ${ }^{17,27}$ and to offer improved patient care and health outcomes in our health system. ${ }^{21}$

\section{Limitations}

The study is focused on a single setting with its unique contextual variances and thus imposes restrictions in generalisability of findings. However, the study can be repeated in varied healthcare contexts and with other healthcare professionals involved in the rehabilitation of PLHIV.

Conflicts of interest - No potential conflict of interest was reported by the authors.

\section{ORCID}

Stacy Maddocks (D) http://orcid.org/0000-0003-2306-7681

Verusia Chetty (iD http://orcid.org/0000-0003-2934-8687

\section{References}

1. World Health Organisation (WHO). 2017 Global health observatory (GHO) data. 2017 [cited 2017 June]. Available from: https://www.who. int/gho/hiv/en/.

2. Shisana O, Rehle T, Simbayi LC, et al. South African national HIV prevalence, incidence and behaviour survey. Cape Town 2012: HSRC Press;2014.

3. Hanass-Hancock J, Regondi I, Egeraat L, et al. HIV-related disability in HIV hyper-endemic countries: A scoping review. World J AIDS 2013;03(03):257. https://doi.org/10.4236/wja.2013.33034

4. ChettyV,MaharajSS. Collaboration between health professionals in the era of antiretroviral therapy.J Assoc Nurs AIDS Care. 2013;24(2):166-75. https://doi.org/10.1016/j.jana.2012.04.005

5. Jelsma J, Mielke J, Powell G, et al. Disability in an urban black community in Zimbabwe. Disabil Rehabil. 2002;24(16):851-9. https://doi.org/10.1080/09638280210129766

6. Brown J, Lewis L, Ellis K, et al. Conflict on interprofessional primary health care teams-can it be resolved? J Interp Care. 2011;25(1):4-10. https://doi.org/10.3109/13561820.2010.497750

7. Baxter SK, Brumfitt SM. Professional differences in interprofessional working. J Interp Care. 2008;22(3):239-51. https://doi.org/10.1080/13561820802054655

8. Cobbing S, Chetty V, Hanass-Hancock J, et al. The essential role of physiotherapists in providing rehabilitation services to people living with HIV in South Africa. South African Journal of Physiotherapy 2013;69(1):22-5.

9. Creswell JW, Poth CN. Qualitative inquiry and research design: Choosing among five approaches. Thousand Oak: Sage publications;2017.

10. Cobbing S, Hanass-Hancock J, Deane M. Physiotherapy rehabilitation in the context of HIV and disability in KwaZulu-Natal, South Africa. Disabil Rehabil. 2014;36(20):1687-94. https://doi.org/10.3109/09638 288.2013.872199

11. Chetty V, Hanass-Hancock J. Development of a model of care for rehabilitation of people living with HIV in a semirural setting in South Africa. JMIR Res Protoc. 2014;3(4):e68. doi:10.2196/resprot.3580.

12. Clarke V, Braun V. Thematic analysis. In: Encyclopedia of critical psychology. New York: Springer;2014. p. 1947-1952.

13. LincolnYS, Guba EG. Butisitrigorous?Trustworthiness and authenticity in naturalistic evaluation. New Direct Eval. 1986;1986:73-84. https://doi.org/10.1002/(ISSN)1551-2371 
14. World Health Organization. Towards a common language for functioning, disability and health ICF. Geneva: World Health Organization; 2002.

15. Rossouw TM. HIV management in practice: South Africa has the largest HIV epidemic in the world and it is therefore imperative that all health care workers are knowledgeable about the management of HIV/AIDS. Contin Med Edu. 2011;29(5):200-202CME.

16. Bechard DJ, Day AM, Dufour SP, et al. How medical students conceptualize disability: Implications for interprofessional practice and education. J Res Interprof Prac Edu. 2010;1(2):159-78.

17. Chetty V, Hanass-Hancock J. The need for a rehabilitation model to address the disparities of public healthcare for people living with HIV in South Africa: opinion papers. Afr J Disabil. 2015;4(1):1-6.

18. Perreault K, Dionne CE, Rossignol M, et al. Interprofessional practices of physiotherapists working with adults with low back pain in Québec's private sector: Results of a qualitative study. BMC Musculoskelet Disord. 2014;15(1):1023. https://doi.org/10.1186/1471-2474-15-160

19. Curran VR, Sharpe D, Flynn K, et al. A longitudinal study of the effect of an interprofessional education curriculum on student satisfaction and attitudes towards interprofessional teamwork and education. J Interprof Care. 2010;24(1):41-52. https://doi.org/10.3109/13561820903011927

20. D'amour D, Oandasan I. Interprofessionality as the field of interprofessional practice and interprofessional education: An emerging concept. J Interprof Care. 2005;19(sup1):8-20. https://doi.org/10.1080/13561820500081604
21. D'Eon M, Proctor P, Cassidy J, et al. Evaluation of an interprofessional problem-based learning module on care of persons living with HIV/ AIDS. J Res Interprof Pract Edu. 2010;1(2):109-26.

22. Miller $\mathrm{KL}$, Reeves $\mathrm{S}$, Zwarenstein $\mathrm{M}$, et al. Nursing emotion work and interprofessional collaboration in general internal medicine wards: a qualitative study. J Adv Nurs. 2008;64(4):332-43. https://doi.org/10.1111/jan.2008.64.issue-4

23. Rose MA, Smith K, Veloski JJ, et al. Attitudes of students in medicine, nursing, occupational therapy, and physical therapy toward interprofessional education. J Allied Health. 2009;38(4):196-200.

24. Odebiyi DI, Amazu AR, Akindele MO, et al. Evaluation of the mode of referral of patients for physiotherapy by physicians. Afr J Physiother Rehabil Sci. 2010;2(1):14-20.

25. Cowan MJ, Shapiro M, Hays RD, et al. The Effect of a Multidisciplinary Hospitalist/Physician and Advanced Practice Nurse Collaboration on Hospital Costs. J Nurs Admin. 2006;36(2):79-85. https://doi.org/10.1097/00005110-200602000-00006

26. Zwarenstein M, Goldman J, Reeves S. Interprofessional collaboration: Effects of practice-based interventions on professional practice and healthcare outcomes. Cochrane Database of Syst Rev. 2009; (3):Art. No.: CD000072. doi:10.1002/14651858.CD000072.pub2.

27. Beattie A. War and peace among the health tribes. Interprofessional Relations in Health Care. London: Edward Arnold;1995. p. 11-26.

Received: 26-06-2017 Accepted: 17-09-2017 